Received: $\quad 2019.03 .04$

Accepted: 2019.04.11

Published: 2019.05 .05

\title{
Combined Signature of the Fecal Microbiome and Plasma Metabolome in Patients with Ulcerative Colitis
}

Authors' Contribution: Study Design A Data Collection B Statistical Analysis C Data Interpretation D Manuscript Preparation E Literature Search F Funds Collection G

\author{
ACE 1 Meiling Sun \\ D 1 Bing Du \\ B 2 Yang Shi \\ C 3 Yue Lu \\ F 2 Yangyang Zhou \\ AG 1,2 Bingrong Liu
}

\author{
1 Department of Gastroenterology and Hepatology, The Second Affiliated Hospital \\ of Harbin Medical University, Harbin, Heilongjiang, P.R. China \\ 2 Department of Gastroenterology and Hepatology, The First Affiliated Hospital of \\ Zhengzhou University, Zhengzhou, Henan, P.R. China \\ 3 Department of Gastroenterology and Hepatology, The First Affiliated Hospital of \\ Harbin Medical University, Harbin, Heilongjiang, P.R. China
}

Corresponding Author: Source of support:

Background: Ulcerative colitis is a chronic, idiopathic inflammatory disease that destroys the colon structure. Nevertheless, the exact pathogenesis is not clear and needs to be fully elucidated.

Material/Methods: Stool and plasma samples were used for 165 ribosomal RNA sequencing and liquid chromatography mass spectrometry, respectively. In addition, we detected the level of trimethylamine N-oxide. Finally, we performed Pearson correlation analysis between the microbiome and the metabolome.

Results: Twenty-three active ulcerative colitis, 25 inactive ulcerative colitis, and 30 control cases were included. Thirtyfour significantly different metabolites were found between the active ulcerative colitis and control groups, 38 were found between the inactive ulcerative colitis and control groups, and only 1 was found between the active ulcerative colitis and inactive ulcerative colitis groups. The plasma trimethylamine $\mathrm{N}$-oxide level of the inactive ulcerative colitis and active ulcerative colitis groups was significantly higher than that of the control group. Moreover, we identified significant changes in 24, 18, and 12 bacterial genera for active ulcerative colitis-control, inactive ulcerative colitis-control, and active ulcerative colitis-inactive ulcerative colitis, respectively. Cross-correlation indicated an association between sphingosine 1-phosphate and Roseburia, Klebsiella, and Escherichia-Shigella. Through the pathway analysis, we found sphingolipid metabolism was one of the most significantly increased pathways.

Conclusions: Although levels of trimethylamine N-oxide were higher in ulcerative colitis patients, they did not achieve statistical significance in active ulcerative colitis and inactive ulcerative colitis groups. Sphingosine 1-phosphate was increased in ulcerative colitis patients and there were several microbiota associated with it. Although further study is still needed, sphingosine 1-phosphate will probably become a new target for treatment of ulcerative colitis.

\section{MeSH Keywords:}

Colitis, Ulcerative $\bullet$ Metabolome • Microbiota • Receptors, Lysosphingolipid

Full-text PDF: https://www.medscimonit.com/abstract/index/idArt/916009

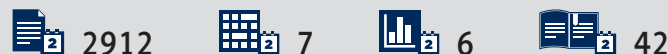




\section{Background}

Inflammatory bowel disease (IBD) involves chronic, idiopathic, and relapsing inflammation in the gastrointestinal (GI) tract, including Crohn's disease (CD) and ulcerative colitis (UC) [1]. UC is characterized by superficial mucosal inflammation, rectal bleeding, diarrhea, and abdominal pain. There are about 3.7 million people with IBD in Europe and North America [2-4], and the incidence of IBD has been increasing in Asian countries such as China [5], India, and South Korea [2,6] over the past 2 decades. In general, $U C$ is more prevalent than $C D$, with a higher incidence [7]. Researchers gradually have discovered that $U C$ is a very complicated disease that involves heredity, environment, microbiology, and abnormal immune system function [8-10]. However, the exact pathogenesis of UC remains unclear and effective therapeutic methods for the disease are lacking.

Some nutrients containing specific trimethylamine (TMA), such as phosphatidylcholine, choline, and carnitine, can provide a carbon fuel source for gut microbes, and TMA is then transported to the liver, where it can be rapidly converted into TMA N-oxide (TMAO) [11]. Multiple studies have shown that plasma levels of TMAO are associated with inflammation, such as priming NLRP3 inflammasome [12] and nuclear factor-KB [13]. However, the plasma levels of TMAO are lowed in IBD, and TMAO levels are lower in inflammatory bowel disease, including ulcerative colitis and Crohn's disease [14]. The present study assessed the level of TMAO only in ulcerative colitis and explored whether the change in TMAO would be different.

Many studies have reported dysbiosis in the GI microbiomes of IBD patients [15-18], thus emphasizing the important effects of the intestinal microbiota in health individuals and in people with IBD. Intestinal bacteria also affect the pathogenesis of IBD through their metabolites [19].

The present study used both metabolome and microbiome methods to assess diversities in samples from healthy controls (Control), inactive UC (iUC), and active UC (aUC) groups. In addition, we defined the level of TMAO in UC. Finally, we investigated correlations between the metabolites and bacteria. We performed this study hoping to find different metabolites and bacteria in ulcerative colitis patients compared with healthy people, and explored the role of metabolites and bacteria in the developmental mechanism of ulcerative colitis. Our results may lead to identification of new targets for the treatment of ulcerative colitis.

\section{Material and Methods}

\section{Subjects}

All patients and healthy persons were recruited from the Second Affiliated Hospital of Harbin Medical University. Patients were recruited after being diagnosed by experienced gastroenterologists without any therapies using 5 -aminosalicylic acid, steroids, or biologics. Exclusion criteria for all patients with UC were pregnancy or underlying malignancies, or using antibiotics in the 4 weeks before sample collection. The healthy volunteers were recruited locally, and had no history of a gastrointestinal or metabolic disease and had not used antibiotics or any other medicine influencing intestinal microbiota in the last 4 weeks. Ethics approval was granted by the Ethics Committee of Harbin Medical University. Prior written patient informed consents were obtained from all subjects.

At the time of inclusion, disease activity was assessed in the UC patients using the UC Disease Activity Index (UCDAI) [20], which includes 4 variables: (a) stool frequency, (b) rectal bleeding, (c) appearance of mucosa, and (d) physician's assessment of disease severity. Each variable has different values for severity, with 0 representing normal and 3 representing the most severe. aUC was defined as a UCDAI $\geq 6$ and iUC was defined as a UCDAI <6. Table 1 provided clinical details.

Plasma and stool samples were collected from persons with confirmed aUC $(n=23)$ and $\mathrm{IUC}(\mathrm{n}=25)$ and healthy control $(n=30)$ subjects, respectively. Fecal samples were collected before starting bowel cleansing. Blood sample was collected into ethylene diamine tetraacetic acid (EDTA) tubes and immediately centrifuged at $1500 \mathrm{~g}$ for $10 \mathrm{~min}$ to obtain plasma. Samples were collected between July 2016 and March 2017 and were stored at $-80^{\circ} \mathrm{C}$ until analyzed.

\section{Library Preparation and Illumina Mi-Seq Sequencing}

Next-generation sequencing library preparation and Illumina Mi-Seq sequencing were performed with Smart Nuclide (Suzhou, China). DNA samples were quantified using a Qubit 3.0 Fluorometer. A total of 30-50 ng DNA was used to generate amplicons by targeting the $\mathrm{V} 3$ and $V 4$ hypervariable regions of prokaryotic $16 \mathrm{~S}$ ribosomal DNA (16S rDNA) using forward primers containing the sequence "CCTACGGRRBGCASCAGKVRVGAAT" and reverse primers containing the sequence "GGACTACNVGGGTWTCTAATCC".

\section{S Ribosomal RNA (16S rRNA) Data Analysis}

To analyze 16S rRNA data, we used the QIIME data analysis package. Sequences were grouped into operational taxonomic units (OTUs). Alpha diversity indexes were calculated using the 
Table 1. Characteristics of UC patients and healthy controls.

\begin{tabular}{|c|c|c|c|}
\hline \multirow{2}{*}{ Characteristics } & \multicolumn{2}{|c|}{ Ulcerative colitis } & \multirow{2}{*}{ Control $\mathbf{N}=\mathbf{3 0}$} \\
\hline & aUC $\mathrm{N}=23$ & iUC $N=25$ & \\
\hline Gender (Male/Female) & $12 / 11$ & $8 / 17$ & $15 / 15$ \\
\hline Age, years (mean, range) & $46(18-72)$ & $47(20-73)$ & $41(19-66)$ \\
\hline \multicolumn{4}{|l|}{ Disease extent } \\
\hline E1 proctitis (\%) & $(13)$ & $(48)$ & \\
\hline E2 left-sided colitis (\%) & $2 \quad(8.7)$ & $(32)$ & \\
\hline E3 pancolitis (\%) & $18 \quad(78.3)$ & $(20)$ & \\
\hline DAI (mean, range) & $6.3 \quad(6-11)$ & $4.4 \quad(3-5)$ & \\
\hline Smoking (\%) & $8 \quad(34.8)$ & $(24)$ & (30) \\
\hline
\end{tabular}

DAI - disease activity index; aUC - active ulcerative colitis; iUC - inactive ulcerative colitis.

Shannon index for diversity and the Chao1 index for richness. Metastats analysis was conducted to investigate differences in the relative abundance for each genus among the Control, iUC, and aUC groups. $P$ value less than 0.05 was used to define significant genus differences.

\section{Plasma preparation}

The prepared sample extracts were filtered through a 0.22- $\mu \mathrm{m}$ membrane. For quality control (QC) samples, $20 \mu \mathrm{L}$ from each prepared sample extract was pooled. These QC samples were used to monitor deviations caused by the pooled mixtures and were compared to errors caused by instrumentation. The remaining samples were used for liquid chromatography mass spectrometry (LC-MS) testing.

\section{Metabolomics analyses}

All samples were analyzed in a double random order: the first step was the metabolite extraction phase, and the second step was liquid chromatography injection. Chromatographic separation was performed on an Acquity UPLC system with an ACQUITY UPLC BEH C18 $(100 \times 2.1 \mathrm{~mm}, 1.7 \mu \mathrm{m}$, Waters $)$ column at a temperature of $40^{\circ} \mathrm{C}$. Then, an equilibrated sample $(10 \mu \mathrm{L})$ was injected into the column. The electron spray ionization mass spectrometry (ESI-MS) experiments were carried out on a Thermo LTQ-Orbitrap XL mass spectrometer with spray voltage of $4.8 \mathrm{kV}$ in positive mode and $4.5 \mathrm{kV}$ in negative mode. Investigators were blinded to the patient information when performing these analyses.

\section{Bioinformatic analysis of metabolomics}

First, the raw LC-MS data were converted into $\mathrm{mzXML}$ file format and then processed by the XCMS tool box. Second, the data were arranged in a data matrix consisting of the mass to charge ratio $(\mathrm{m} / \mathrm{z})$, retention time, and peak area. Third, the output of the XCMS was further processed to analyze the subsequent results using Microsoft Excel.

\section{Trimethylamine $\mathrm{N}$-oxide (TMAO), choline, betaine, creatinine, and L-carnitine measurements}

Plasma concentrations of TMAO, choline, betaine, creatinine, and L-carnitine were measured by LC-MS. Plasma samples $(20 \mu \mathrm{L})$ were precipitated by adding $750 \mu \mathrm{L}$ of acetonitrile (ACN) and $10 \mu \mathrm{L}$ internal standards (TMAO-d9, choline- $\mathrm{d} 9$, betaine- $\mathrm{d}$ 9, creatinine-d3, and L-carnitine-d3). Chromatographic separation was performed using an ACQUITY UPLC BEH HILIC device $(100 \times 2.1 \mathrm{~mm}, 1.7 \mu \mathrm{m}$, Waters). The mass spectrometer ( $A B$ 4000 ) with a heated electrospray ionization source was set in positive mode to detect the amount of TMAO.

\section{Construction of metabolic pathways}

The construction, interaction, and pathway analyses of potential metabolites were performed with MetPA, and the Kyoto Encyclopedia of Genes and Genomes (KEGG). Metabolic pathway analysis was performed with MetPA by filtering the dataset using an FDR-adjusted $P$ value $<0.05$ and impact value $>0.1$ to reveal how significant metabolites are involved in different pathways.

\section{Statistical analysis}

Multivariate statistical analysis was performed. We used principal component analysis (PCA), partial least squares discriminant analysis (PLS-DA), and orthogonal projections to latent structures discriminant analysis (OPLS)-DA to assess the data. The significance test of the Pearson's correlation coefficient was carried out to determine metabolites with significant different microbiota between groups. All statistical analyses were 


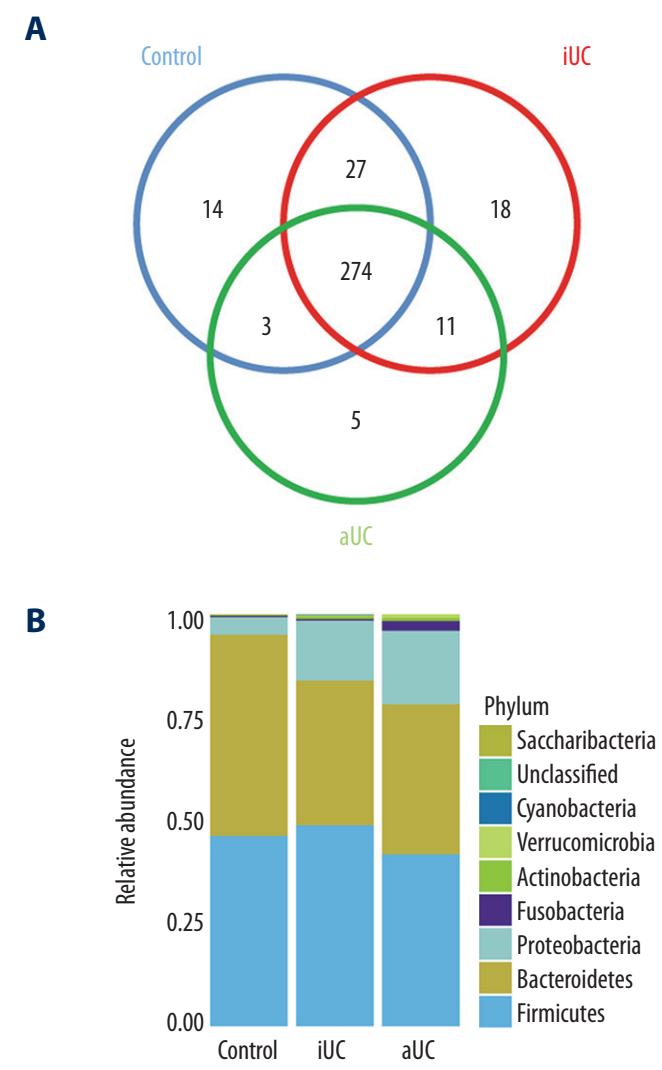

C

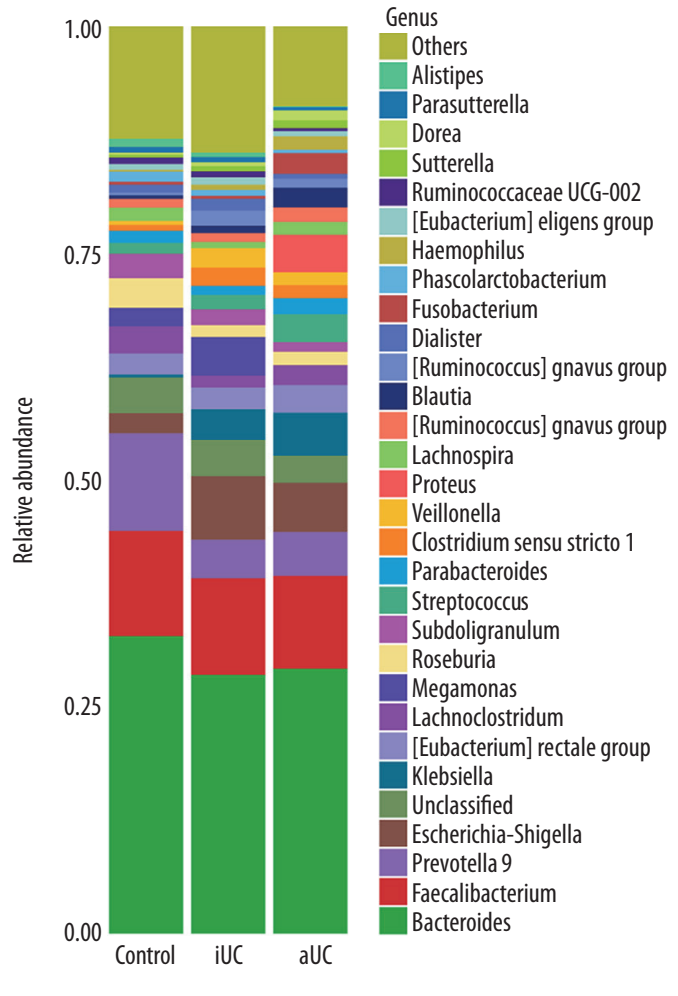

Figure 1. aUC, iUC, and Control patients harbor distinct microbial populations. (A) The number of OTUs present in aUC patients only (green), iUC patients only (red), Control patients only (blue), or shared among the 3 groups in a scaled Venn diagram. (B) Relative abundance at the phylum level. (C) Relative abundance at the genus level. Different phyla and genera are colorcoded. aUC - active ulcerative colitis; iUC - inactive ulcerative colitis.

performed using R software. $P$ value $<0.05$ was considered to indicate a significant difference.

\section{Results}

\section{$16 S$ rRNA data analysis of microbiota}

A total of 9597636 sequencing reads were obtained from 78 stool samples. The numbers of OTUs varied from 30185 to 65 179. After OTU picking and chimera checking, the total sequencing reads were rarefied to 4798818 . The 3 groups shared a large degree of community similarity after defining OTUs at a level of $3 \%$ dissimilarity. There were 274 OTUs shared by the 3 groups, 301 OTUs were shared between the iUC and Control groups, 277 were shared between the aUC and Control groups, and 285 were shared between the aUC and iUC groups (Figure 1A). The relative abundances of OTUs in the 3 groups at the phylum level are shown for those with an abundance of at least $0.1 \%$ (Figure 1B). Firmicutes, Bacteroidetes, and Proteobacteria were the most abundant phyla in the aUC, iUC, and Control groups, respectively. In the aUC group compared to the Control group, Proteobacteria and Fusobacteria were increased, and Firmicutes and Bacteroidetes decreased. For the iUC group compared to the Control group, the same evidence of variation was found between the groups, except that Firmicutes were increased. However, comparison of the aUC and iUC groups showed more Proteobacteria, Fusobacteria, and Bacteroidetes in the aUC group, with only Firmicutes decreasing.

We also assessed the relative abundances and frequencies of OTUs at the class (Figure 2A), order (Figure 2B), family (Figure 2C), and species, (Figure 2D) levels. At the genus level, Klebsiella, Escherichia-Shigella, Streptococcus, and Blautia were increased in the aUC group, whereas Bacteroides, Faecalibacterium, and Prevotella_9 were all decreased, and the same was found in the iUC group (Figure 1C).

Alpha diversity analysis using the Chao 1 index (Figure $3 \mathrm{~A}$ ) and the Shannon index revealed lower richness for the fecal microbiota of the IUC and aUC groups. Notable differences were observed concerning Chao 1 index and Shannon indexes between 


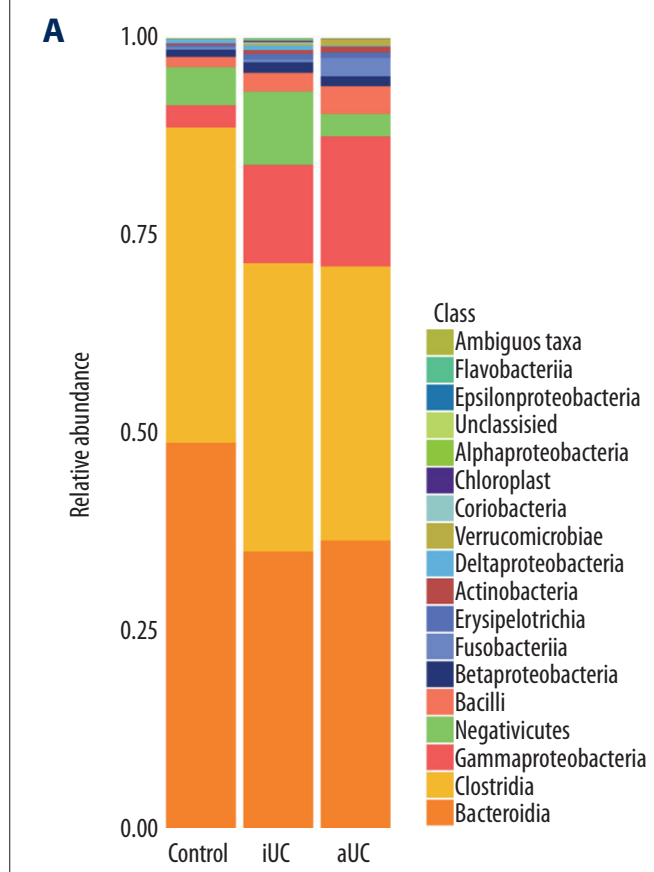

C

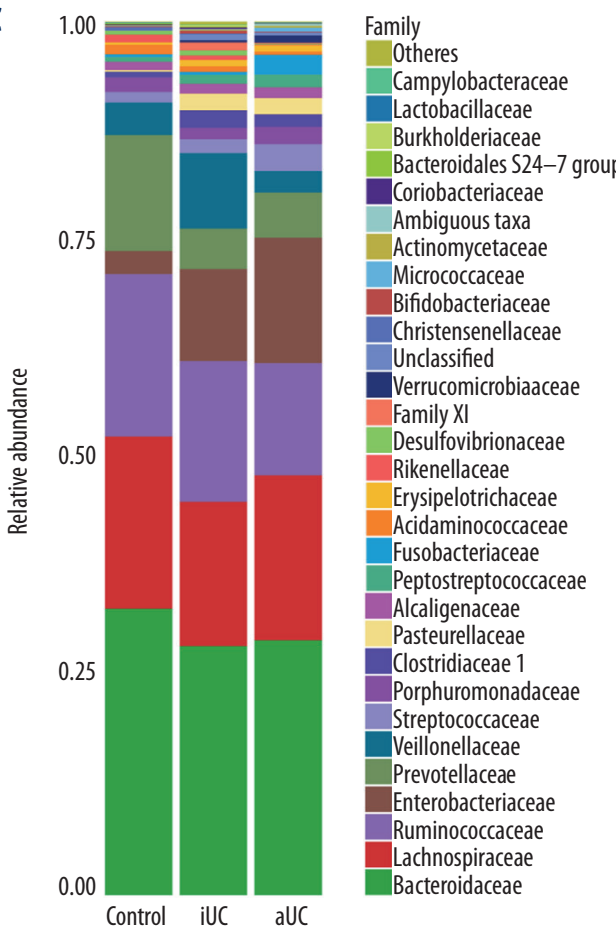

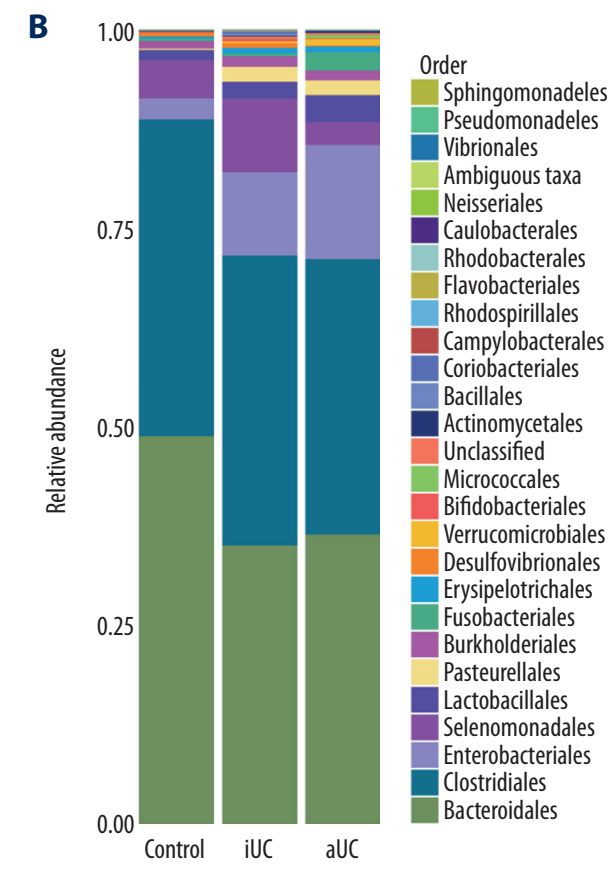

D

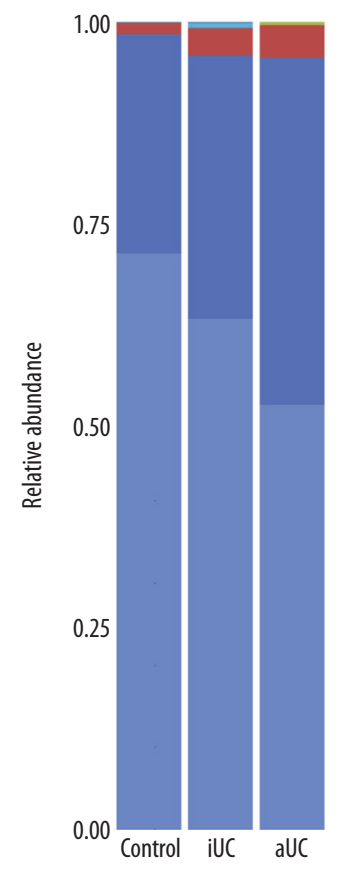

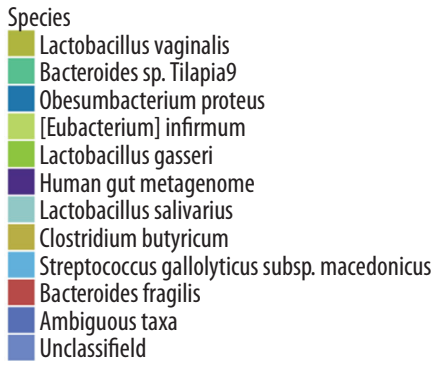

Figure 2. (A) Relative abundance at the class level. (B) Relative abundance at the order level. (C) Relative abundance at the family level. (D) Relative abundance at the species level. Different class, order, family, and species are color-coded. aUC - active ulcerative colitis; iUC - inactive ulcerative colitis. 
A

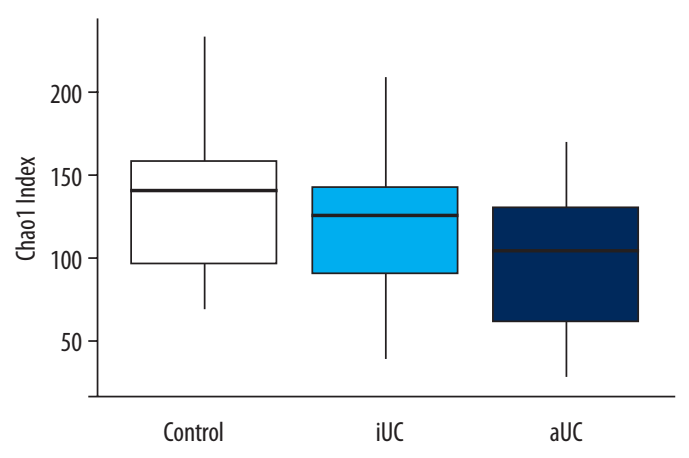

C

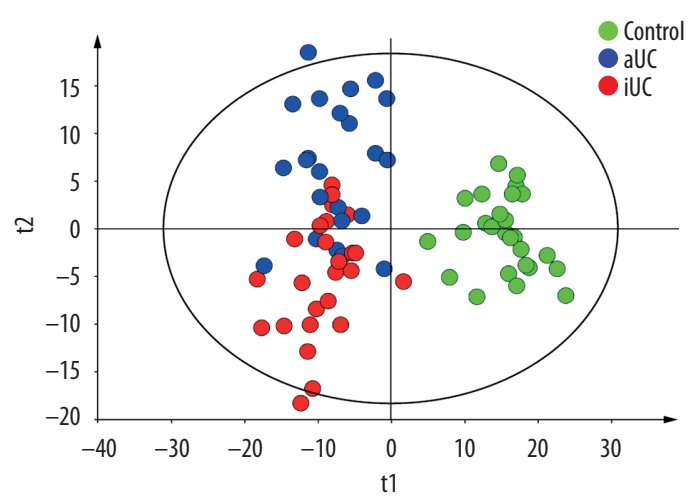

B

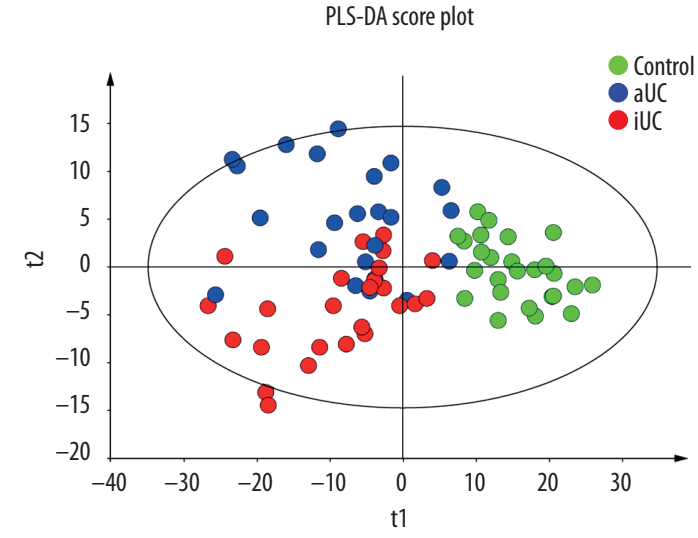

D

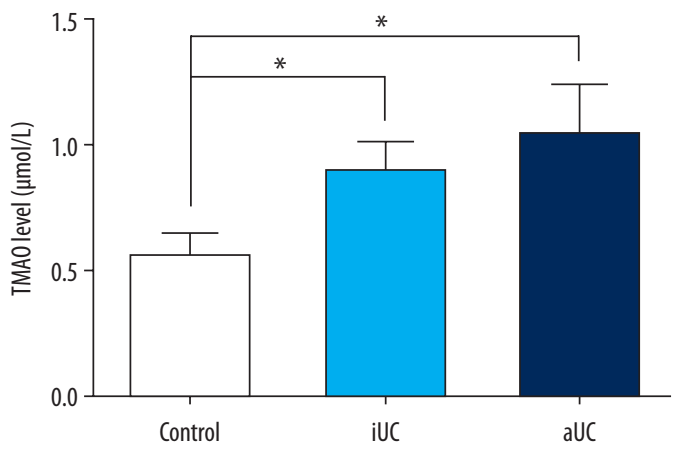

Figure 3. (A) Alpha diversity was shown analyzed by the Chao1 index. Minimum values (the lowest of the line), median values (thick horizontal line), $25^{\text {th }}$ and $75^{\text {th }}$ percentile values (box outline), and maximum values (the highest of the line). (B) PLS-DA and (C) OPLS-DA scores plots based on the metabolite profiling data obtained from Control, iUC and aUC patients, the green, red and blue circles indicate Control, iUC and aUC patients respectively. (D) TMAO levels of the Control, iUC and aUC patients. * Indicates $p<0.05$.

Table 2. Sequencing data with richness and diversity estimation of bacteria taxa in Control, iUC, and aUC groups.

\begin{tabular}{|c|c|c|c|}
\hline Variables & Control $(n=30)$ & iUC $(n=25)$ & aUc $(n=23)$ \\
\hline Chao1 & $135.2 \pm 38.6$ & $124.0 \pm 447.8$ & $100.0 \pm 44.6$ \\
\hline Shannon & $3.95 \pm 0.86$ & $3.83 \pm 0.93$ & $3.34 \pm 1.06$ \\
\hline Simpson & $0.85 \pm 0.1$ & $0.84 \pm 0.09$ & $0.79 \pm 0.19$ \\
\hline Coverage & 0.99 & 0.99 & 0.99 \\
\hline
\end{tabular}

aUC - active ulcerative colitis; iUC - inactive ulcerative colitis.

the gut microbiota of the aUC and Control groups (Table 2). Furthermore, significant $(P<0.05)$ changes in 24 and 18 bacterial genera were revealed, mainly in Metastats-based comparison of the aUC-Control (Table 3) and iUC-Control (Table 4) groups, respectively. There were 10 common genera. Metastatsbased comparison of the aUC and iUC groups revealed significant changes in 12 bacterial genera (Table 5).

\section{Multivariate data analysis of LC-MS spectra}

PLS-DA models comparing 3 groups were generated, and good separation was achieved (Figure 3B). To uncover those metabolic changes with differential power, the O-PLS-DA strategy was subsequently applied to each model, again with clear separation of the 3 groups (Figure 3C). 
Table 3. Significant changes of bacterial genera mainly in the metastats-based comparison of Control-aUC groups.

\begin{tabular}{|c|c|c|c|}
\hline Taxon & Control-mean & aUC-mean & P value \\
\hline Akkermansia & $8.99 \mathrm{E}-05$ & 0.008237 & $1.24 \mathrm{E}-07$ \\
\hline Prevotella_2 & 0.007809 & $4.35 \mathrm{E}-06$ & $4.02 \mathrm{E}-06$ \\
\hline Ruminococcaceae_UCG-014 & 0.006546 & $3.48 \mathrm{E}-05$ & $1.26 \mathrm{E}-05$ \\
\hline Alistipes & 0.00898 & 0.00093 & $3.24 \mathrm{E}-05$ \\
\hline Aggregatibacter & $3.34 \mathrm{E}-06$ & 0.003686 & 0.001249 \\
\hline Rothia & 0.000417 & 0.004357 & 0.001563 \\
\hline Hungatella & 0.006947 & 0.001339 & 0.001634 \\
\hline Lachnospiraceae_NK4A136_group & 0.00768 & 0.001521 & 0.002771 \\
\hline [Ruminococcus]_gnavus_group & 0.003023 & 0.009782 & 0.002961 \\
\hline Tyzzerella_4 & 0.002867 & 0.009589 & 0.002961 \\
\hline [Eubacterium]_coprostanoligenes_group & 0.004443 & 0.000287 & 0.005474 \\
\hline Ruminococcaceae_UCG-005 & 0.003079 & 0.000152 & 0.006475 \\
\hline Sutterella & 0.003067 & 0.008644 & 0.007515 \\
\hline Christensenellaceae_R-7_group & 0.003832 & 0.000404 & 0.009551 \\
\hline Megamonas & 0.020465 & $4.35 \mathrm{E}-06$ & 0.00999 \\
\hline Blautia & 0.00388 & 0.021667 & 0.010989 \\
\hline Phascolarctobacterium & 0.010957 & 0.003805 & 0.011988 \\
\hline Erysipelatoclostridium & 0.0002 & 0.003296 & 0.012848 \\
\hline Mitsuokella & 0.00033 & 0.003598 & 0.012848 \\
\hline Subdoligranulum & 0.027023 & 0.010703 & 0.017982 \\
\hline Paraprevotella & 0.004396 & 0.001004 & 0.018814 \\
\hline Dorea & 0.002254 & 0.0107 & 0.033966 \\
\hline Turicibacter & 0.000623 & 0.002913 & 0.04619 \\
\hline Ambiguous_taxa & 0.000307 & 0.002474 & 0.047841 \\
\hline
\end{tabular}

aUC - active ulcerative colitis; Bold means increasing and italic means decreasing in the aUC group.

Thirty-four metabolites were found to be important for the separation of the aUC group from the Control group (Table 6). Of these 34, 33 metabolites were significantly higher in aUC patients, and only 1 metabolite was significantly decreased in aUC patients.

In comparison between iUC and Control groups, 38 metabolites were able to discriminate between these 2 groups, only 1 metabolite was significantly decreased in iUC patients, whereas 37 were significantly increased (Table 7). Furthermore, 29 common metabolites were revealed by aUC-Control and iUCControl comparison. All were increased in the disease groups. However, compared with the iUC group, aUC patients only had significantly higher concentrations of biliverdin.

\section{TMAO}

Using targeted LC-MS, the plasma TMAO level of the iUC and aUC groups was significantly higher than that of the Control group and there was an association between increasing levels of TMAO associated with increasing disease activity, but it did not achieve statistical significance in the aUC and iUC groups (Figure 3D).

\section{Metabolites changes correlated with microbial genera}

To further explore the associations between the plasma metabolome and fecal microbiome, we assessed correlations of different microbiota at the genus level and metabolites. When comparing the UC group and the Control group, the UC-enriched 
Table 4. Significant changes of bacterial genera mainly in the metastats-based comparison of Control-iUC groups.

\begin{tabular}{|c|c|c|c|}
\hline Taxon & Control-mean & iUC-mean & P Value \\
\hline Parvimonas & $6.67 \mathrm{E}-05$ & 0.006306 & $3.23 \mathrm{E}-06$ \\
\hline [Eubacterium]_ruminantium_group & 0.00071 & 0.005966 & 0.000355 \\
\hline Prevotella_2 & 0.007809 & 0.001308 & 0.000486 \\
\hline Erysipelatoclostridium & 0.0002 & 0.004652 & 0.000954 \\
\hline Aggregatibacter & $3.34 \mathrm{E}-06$ & 0.013148 & 0.002997 \\
\hline Gemella & $9.00 \mathrm{E}-05$ & 0.002756 & 0.003991 \\
\hline Akkermansia & $8.99 \mathrm{E}-05$ & 0.002483 & 0.008791 \\
\hline Ruminococcaceae_UCG-014 & 0.006546 & 0.001876 & 0.01404 \\
\hline Escherichia-Shigella & 0.022275 & 0.070176 & 0.016983 \\
\hline Barnesiella & 0.002257 & 0.000184 & 0.018322 \\
\hline Hungatella & 0.006947 & 0.002324 & 0.018675 \\
\hline Roseburia & 0.032509 & 0.013128 & 0.01998 \\
\hline Mitsuokella & 0.00033 & 0.002816 & 0.02705 \\
\hline Christensenellaceae_R-7_group & 0.003832 & 0.000964 & 0.027963 \\
\hline Haemophilus & 0.001976 & 0.005725 & 0.040361 \\
\hline Paraprevotella & 0.004396 & 0.001292 & 0.04199 \\
\hline Burkholderia-Paraburkholderia & 0 & 0.001743 & 0.042632 \\
\hline [Ruminococcus]_gnavus_group & 0.003023 & 0.016526 & 0.04995 \\
\hline
\end{tabular}

iUC - inactive ulcerative colitis. Bold means increasing and italic means decreasing in the iUC group.

Table 5. Significant changes of bacterial genera mainly in the metastats-based comparison of aUC-iUC groups.

\begin{tabular}{|lccc}
\hline Taxon & Group1_mean & Group2_mean & P_Value \\
\hline Parvimonas & 0.006305729 & 0.000156499 & $3.62 \mathrm{E}-05$ \\
\hline [Eubacterium]_ruminantium_group & 0.005966454 & 0.000195593 & $7.09 \mathrm{E}-05$ \\
\hline Megasphaera & 0.006017201 & 0.000378228 & 0.000592034 \\
\hline Rothia & 0.000391966 & 0.004357345 & 0.004684959 \\
\hline Megamonas & 0.042638497 & $4.35 \mathrm{E}-06$ & 0.004995005 \\
\hline [Eubacterium]_coprostanoligenes_group & 0.004575475 & 0.000286888 & 0.006787169 \\
\hline Akkermansia & 0.002482946 & 0.008237196 & 0.007709616 \\
\hline Tyzzerella_4 & 0.003524233 & 0.009588637 & 0.011138402 \\
\hline Alistipes & 0.005000014 & 0.000930138 & 0.013848166 \\
\hline Ruminococcaceae_UCG-005 & 0.002831513 & 0.000152152 & 0.01612694 \\
\hline Bilophila & 0.004404784 & 0.000882664 & 0.023743361 \\
\hline Turicibacter & 0.000276041 & 0.002913007 & 0.032233079 \\
\hline
\end{tabular}

aUC - active ulcerative colitis; iUC - inactive ulcerative colitis. Bold means increasing and italic color means decreasing in the aUC group.

This work is licensed under Creative Common Attribution NonCommercial-NoDerivatives 4.0 International (CC BY-NC-ND 4.0) 
Table 6. Significant changes of metabolites in the aUC group compared with the Control group.

\begin{tabular}{|ccc|}
\hline \multicolumn{1}{c}{ Name } & VIP & P value \\
\hline Sphinganine & 1.64416 & $1.71 \mathrm{E}-08$ \\
\hline PE (16: 0/0: 0) & 1.73187 & $5.36 \mathrm{E}-08$ \\
\hline L-thyroxine & 1.63935 & $8.37 \mathrm{E}-08$ \\
\hline PE (18: 1(9Z)/0: 0) & 1.36454 & $2.77 \mathrm{E}-07$ \\
\hline Gamma-Caprolactone & 1.49274 & $1.77 \mathrm{E}-06$ \\
\hline Taurine & 1.64994 & $1.96 \mathrm{E}-06$ \\
\hline Creatinine & 1.40727 & $9.11 \mathrm{E}-06$ \\
\hline epsilon-Caprolactam & 1.5111 & $1.44 \mathrm{E}-05$ \\
\hline Biliverdin & 1.19447 & $1.44 \mathrm{E}-05$ \\
\hline Sphingosine 1-phosphate & 1.2551 & $2.07 \mathrm{E}-05$ \\
\hline O-Lauroyl-L-Carnitine & 1.23959 & $4.57 \mathrm{E}-05$ \\
\hline Cortisol & 1.41129 & $6.99 \mathrm{E}-05$ \\
\hline 4-Hydroxyphenylacetic acid & 1.32895 & 0.000219 \\
\hline Isobutyryl carnitine & 1.20777 & 0.000349 \\
\hline m-Xylene & 1.27306 & 0.000792 \\
\hline L-proline & 1.01993 & 0.000792 \\
\hline L-tyrosine & 1.03775 & 0.001055 \\
\hline Bol & &
\end{tabular}

\begin{tabular}{|lcc}
\hline \multicolumn{1}{c}{ Name } & VIP & P value \\
\hline L-phenylalanine & 1.19025 & 0.001215 \\
\hline Gamma-Glu-Leu & 1.13083 & 0.001397 \\
\hline Catechol & 1.30834 & 0.001497 \\
\hline N,O-Didesmethylvenlafaxine & 1.20739 & 0.001604 \\
\hline Citric acid & 1.42972 & 0.001838 \\
\hline L-tryptophan & 1.20252 & 0.00225 \\
\hline Melanin & 1.33432 & 0.00225 \\
\hline (2E)-Hexenal & 1.28472 & 0.004043 \\
\hline L-isoleucine & 1.01687 & 0.004307 \\
\hline Keto-D-Fructose & 1.48846 & 0.004586 \\
\hline L-valine & 1.14464 & 0.004882 \\
\hline L-leucine & 1.14401 & 0.005195 \\
\hline Traumatic acid & 1.25265 & 0.007944 \\
\hline Phosphatidylinositol lyso 20: 4 & 1.20515 & 0.010044 \\
\hline L-Glutamine & 1.05434 & 0.01193 \\
\hline (R)-2-Hydroxyoctadecanoic & 1.20776 & 0.014124 \\
\hline acid & 1.14315 & 0.018576 \\
\hline 5-oxo-D-proline & \\
\hline
\end{tabular}

Bold means increasing and italic means decreasing in the aUC group.

Akkermansia displayed a positive correlation with UC-enriched 5-oxo-D-proline, gamma-Glu-Leu, O-lauroyl-L-carnitine, and melanin. Additionally, UC-enriched Erysipelatoclostridium displayed a positive correlation with UC-enriched(2E)-hexenal. The UC-enriched [Ruminococcus]_gnavus_group was also positively correlated with citric acid (Figure 4). When analyzing the aUC and iUC groups, there was no obvious correlation between biliverdin and different genera. To expand such correlations, we combined the altered metabolites with examined genera (Figure 5). From the expanded correlations, we found TMAO was obviously positively correlated with Holdemanella, Ruminococcus_1, Ruminococcaceae_NK4A214_group, and Christensenellaceae_R-7_group. S1P was negatively associated with Roseburia and positively associated with Klebsiella and Escherichia-Shigella.

\section{Pathways analysis}

Metabolic pathways associated with phenylalanine metabolism, tyrosine metabolism, and sphingolipid metabolism between the aUC-Control and iUC-Control groups were noted (Figure 6A, 6B).

\section{Discussion}

There is increasing evidence that in addition to genetics and lifestyle, the intestinal microbiota is an important factor for the development of many diseases. The gut microbiome is a complicated community which is metabolically active and produces many kinds of metabolites that can directly influence the host phenotype [21]. Many researchers also have demonstrated that the intestinal microbiota interacts with UC, which is relevant in characterizing and treating patients [22-24].

To the best of our knowledge, this is the first attempt to examine UC disease activity by integrating the microbiome and metabolome. We present several novel findings. First, we detected an altered microbiota composition and significant microbiota-metabolite relationships. Second, we found significant associations with plasma concentrations of TMAO. Finally, we built metabolic pathways and mapped metabolites that were altered into likely relevant pathways to explain metabolism.

Some alterations of the gut microbiome can help to distinguish UC patients from healthy persons. Our study found that the genus R. gnavus [Ruminococcus_gnavus_group] was enriched in 
Table 7. Significant changes of metabolites in the iUC group compared with the Control group.

\begin{tabular}{|c|c|c|}
\hline Name & VIP & P value \\
\hline Sphinganine & 1.56764 & $3.48 \mathrm{E}-09$ \\
\hline PE (16: 0/0: 0) & 1.56031 & $1.34 \mathrm{E}-08$ \\
\hline L-thyroxine & 1.53382 & $6.06 \mathrm{E}-08$ \\
\hline PE (18: 1(9Z)/0: 0) & 1.08848 & $1.02 \mathrm{E}-07$ \\
\hline Creatinine & 1.3639 & $8.34 \mathrm{E}-07$ \\
\hline Taurine & 1.59564 & $1.01 \mathrm{E}-06$ \\
\hline L-leucine & 1.39231 & $4.89 \mathrm{E}-06$ \\
\hline O-Lauroyl-L-Carnitine & 1.24239 & $6.4 \mathrm{E}-06$ \\
\hline Gamma-Glu-Leu & 1.40981 & $6.99 \mathrm{E}-06$ \\
\hline Sphingosine 1-phosphate & 1.13255 & $1.4 \mathrm{E}-05$ \\
\hline L-phenylalanine & 1.368 & $3.26 \mathrm{E}-05$ \\
\hline m-Xylene & 1.25085 & $3.84 \mathrm{E}-05$ \\
\hline L-tryptophan & 1.37052 & $6.23 \mathrm{E}-05$ \\
\hline Gamma-Caprolactone & 1.35019 & $6.74 \mathrm{E}-05$ \\
\hline Pipecolic acid & 1.13348 & $6.74 \mathrm{E}-05$ \\
\hline epsilon-Caprolactam & 1.32018 & $9.24 \mathrm{E}-05$ \\
\hline $\begin{array}{l}\text { 3-Hydroxy-3-Methylglutaric } \\
\text { acid }\end{array}$ & 1.08923 & 0.000117 \\
\hline L-valine & 1.31087 & 0.000147 \\
\hline L-proline & 1.0628 & 0.000411 \\
\hline
\end{tabular}

\begin{tabular}{|lcc|}
\hline \multicolumn{1}{c}{ Name } & VIP & P value \\
\hline Amidotrizoic acid & 1.06035 & 0.000508 \\
\hline L-tyrosine & 1.07928 & 0.000882 \\
\hline Citric acid & 1.41072 & 0.000882 \\
\hline L-isoleucine & 1.14751 & 0.001079 \\
\hline Melanin & 1.64956 & 0.001818 \\
\hline Cortisol & 1.02395 & 0.0022 \\
\hline Hydroxyphenyllactic acid & 1.51524 & 0.003001 \\
\hline L-Glutamine & 1.17157 & 0.00339 \\
\hline N,O-Didesmethylvenlafaxine & 1.04363 & 0.003601 \\
\hline Azelaic acid & 1.1804 & 0.008576 \\
\hline 4-Hydroxyphenylacetic acid & 1.00724 & 0.010112 \\
\hline L-(-)-3-Phenyllactic acid & 1.20054 & 0.010112 \\
\hline (R)-2-Hydroxyoctadecanoic & 1.14961 & 0.010676 \\
\hline acid & 1.02159 & 0.029535 \\
\hline 5-oxo-D-proline & 1.00253 & 0.048952 \\
\hline Indolelactic acid & & \\
\hline Keto-D-Fructose & 1.32524 & 0.018045 \\
\hline Uric acid & 1.20529 & 0.019964 \\
\hline (2E)-Hexenal & & 0.013221 \\
\hline 4-Methyl-2-oxopentanoic acid & & 0.013221 \\
\hline
\end{tabular}

Bold means increasing and italic means decreasing in the iUC group.

UC patients; it expresses $\beta$-glucuronidase activity that can produce harmful metabolites in the colon, which can lead to local inflammation [25]. The other enriched Erysipelatoclostridium is considered an opportunistic pathogen in the human intestine [26]. The beneficial taxon Christensenellaceae can produce butyric acid, which improves the mucosal barrier function of the colon, regulates the immune system, and has anti-inflammatory properties because it can downregulate pro-inflammatory cytokines [27]. Although the changes in bacteria may only be a reflection of the disease process or can the cause of the disease, we still look forward to finding different bacteria that could be used as biomarkers of ulcerative colitis. In our study, we found some different bacteria in ulcerative colitis, and the significantly changed pathogens identified in this study may be signatures of UC disease, but further study is needed to verify them to be the signature of ulcerative colitis disease.

Gut metabolites are an important link between gut microbes and host biological functions. UC results in dramatic changes in the plasma metabolome. Phosphatidylethanolamines (PEs) are the primary lipid components of the inner bacterial membrane, and an increase in PE levels is indicative of significant cell injury. PEs are also associated with bacterial stress responses [28]. Thyroid hormones regulate cellular signaling pathways, such as intracellular mitogen-activated protein kinase (MAPK 1 and 2) and phosphatidylinositol-3-kinase (PI3K) [29], and indirectly stimulate nitric oxide synthase [30]. Moreover, thyroid hormone can also stimulate the S100A8/MyD88/NF-KB signaling pathway in cardiomyocytes [31]. Sphingolipids are essential constituents of cellular membranes and are involved in cell proliferation, viability, motility, migration, and lymphocyte trafficking as signaling molecules [32]. Sphingosine (the sphingosine 1-phosphate precursor) is derived from the catabolism of endogenous cellular sphingolipids, and sphingosine 1-phosphate (S1P) is the 1-phosphorylated form of sphingosine. S1P is involved in inflammatory-based diseases such as asthma [33], rheumatoid arthritis [34], multiple sclerosis [35], and IBD [36,37]. The cross-correlation between the gut microbiome and the metabolome indicates an association between bacterial communities and functional metabolites. It can be concluded that S1P 


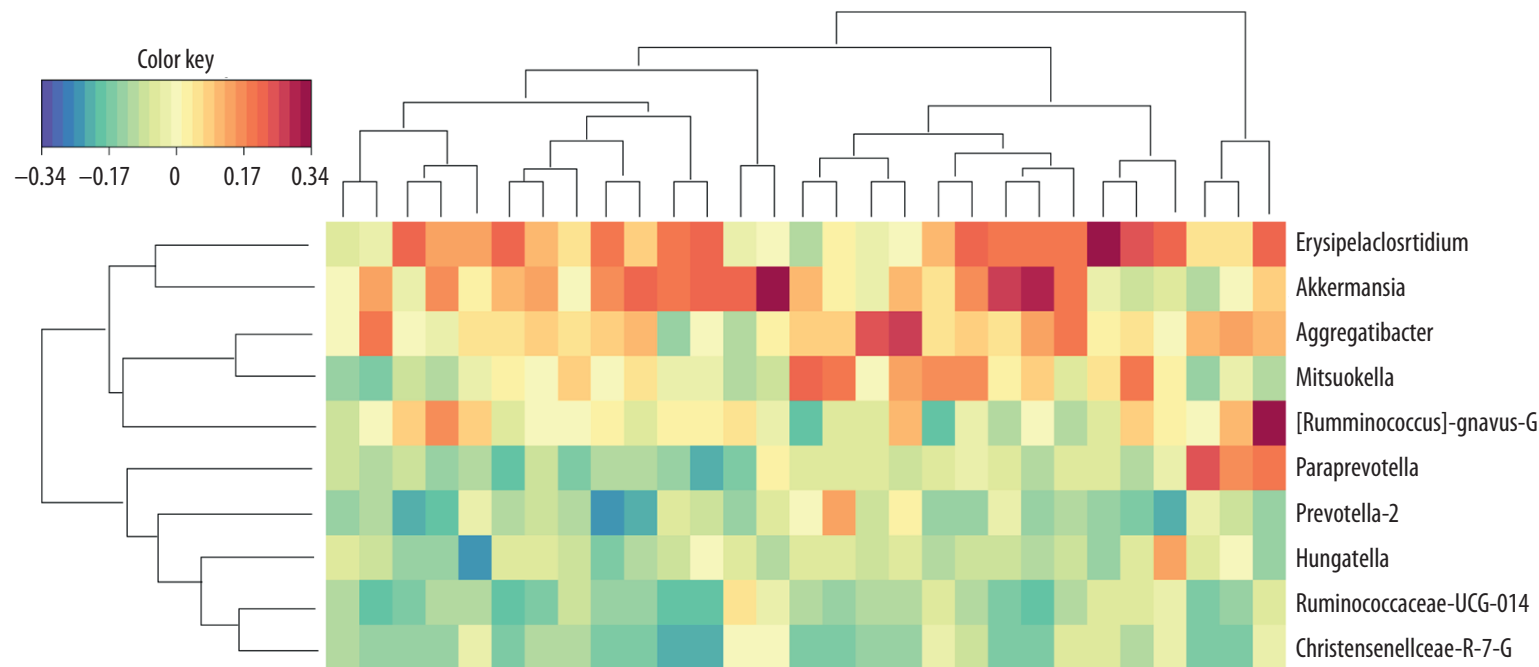

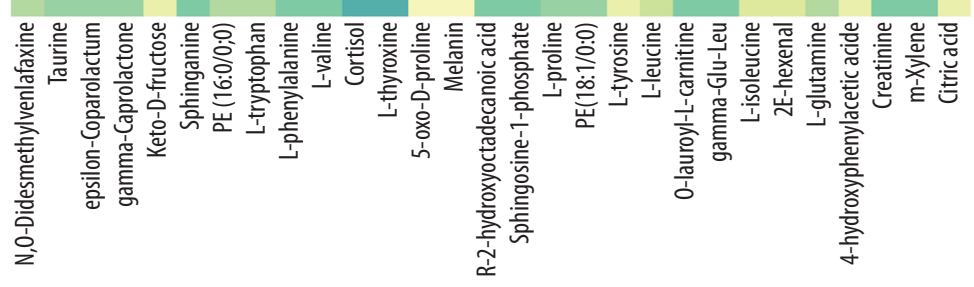

Figure 4. Inter-omic Pearson's correlation between significant different metabolites and bacterial genera for UC and Control patients. The correlation was considered statistically significant with $p<0.05+|\mathrm{r}|$ coefficient $>0.22$.

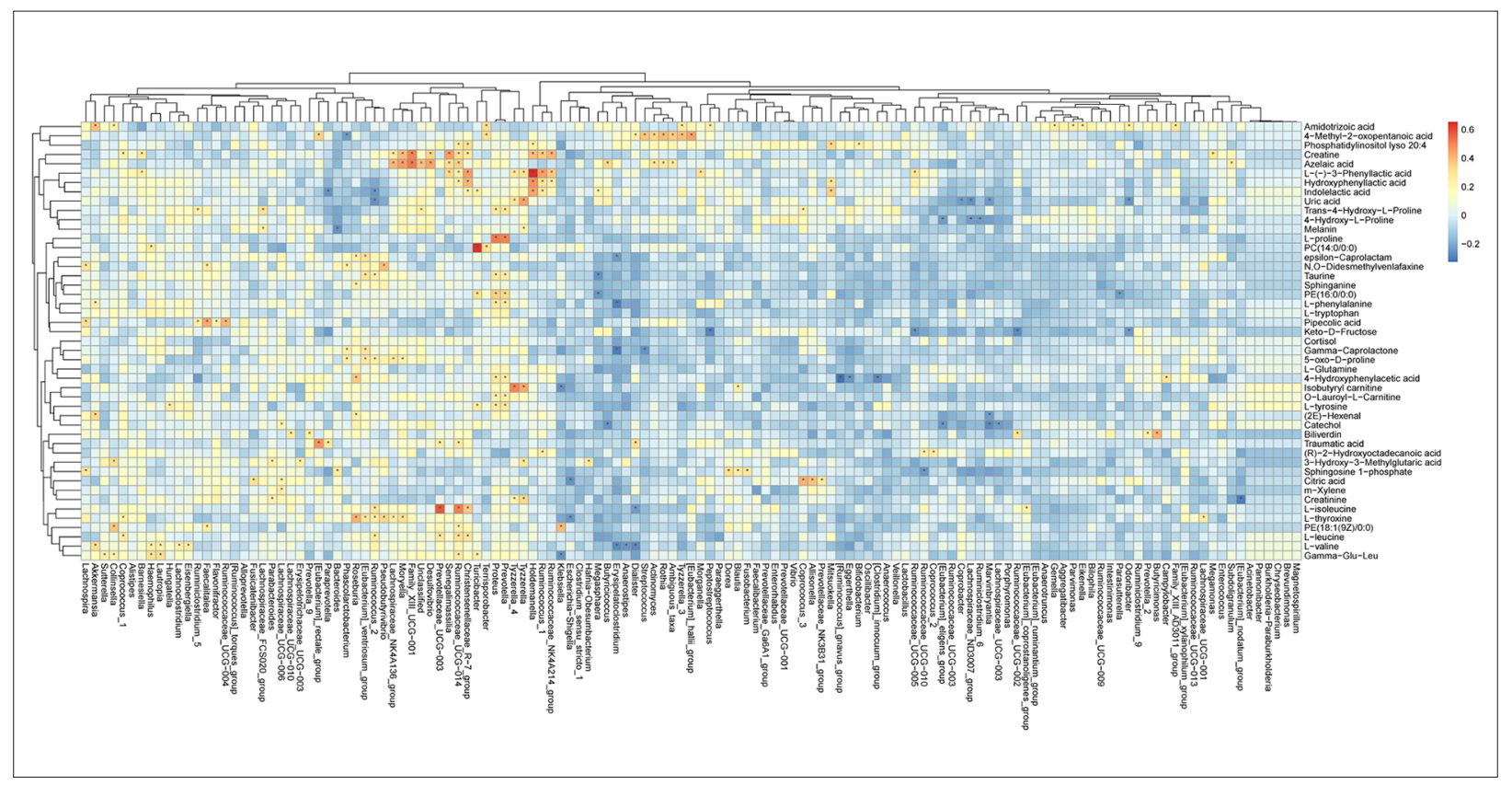

Figure 5. Inter-omic Pearson's correlation between significant different metabolites and all bacterial genera for UC and Control patients. * Indicates levels of significance with $p<0.05$. 
A

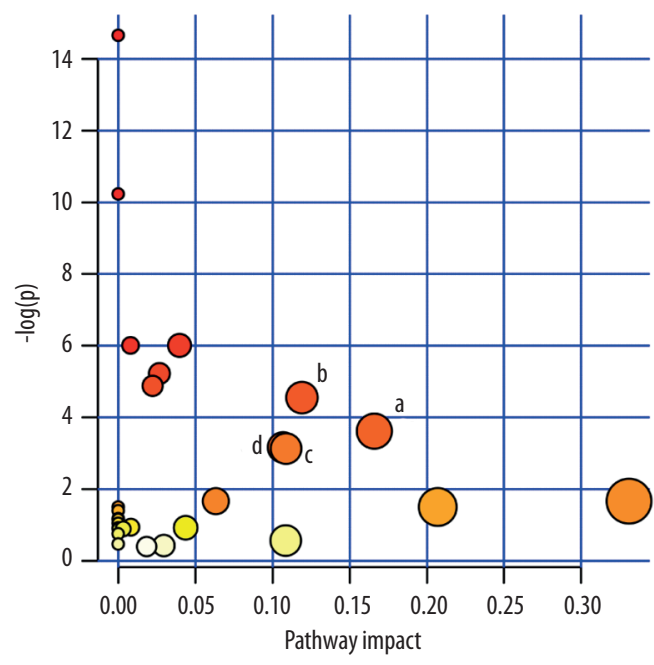

B

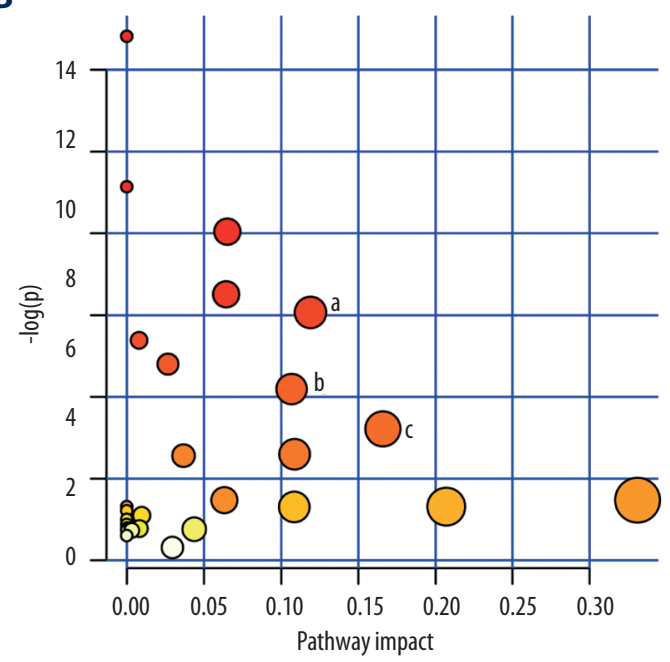

Figure 6. Summary of pathway analysis with MetPA in aUC-Control patients (A). a - Sphingolipid metabolism; b - Phenylalanine metabolism; c - Arginine and proline metabolism; $d$ - Tyrosine metabolism. Another summary of pathway analysis with MetPA in iUC-Control patients (B). a - Phenylalanine metabolism; b - Tyrosine metabolism; c - Sphingolipid metabolism.

is negatively associated with Roseburia and positively associated with Klebsiella and Escherichia-Shigella. Some researchers have reported that a decrease in Roseburia spp. is associated with the gut microbiota of patients with IBD [38] and that Roseburia spp specifically colonizes mucins, which govern mucosal butyrate production [39]. Another research group reported that sodium butyrate can lead to apoptosis of colon cancer cells by influencing sphingosine kinase 2 [40]. While Roseburia spp and butyrate production were decreased, we concluded that decreased butyrate stimulated sphingosine kinase 2 and then increased sphingosine and S1P. The abundance of Escherichia-Shigella (Enterobacteriaceae) was higher in the feces of UC patients and in rectal biopsies from CD patients, indicating a possible association with the pathogenesis of IBD [41,42].

According to the pathway analysis results in the present study, the metabolism of phenylalanine, tyrosine, and sphingolipid was particularly active in UC patients.

In our study, we found that plasma TMAO concentration in the UC group was significantly higher compared to the Control group, and there was also an increasing trend from iUC to aUC groups. In addition, TMAO was obviously positive with 4 genera and they all belonged to Firmicutes. This information shows that TMAO may serve as a biomarker to help UC diagnosis. However, further investigation is needed to assess the value of TMAO for disease activity analysis and to elucidate the relationship between TMAO and those microbiota.
We found little difference between the iUC and aUC groups; only 1 metabolite (biliverdin) was increased significantly in the aUC group and there was no identical correlation between this metabolite and divergent microbiota at the genus level. Considering the small sample sizes and deviation in collecting samples, we found it difficult to assess the disease activity. Limitations of our study include the small sample size and the fact that outcomes were from a single center. Disease activity was assessed in the ulcerative colitis patients using the UC Disease Activity Index, but some items of the scoring system were influenced by researchers' subjective judgements, which might have led to biases.

\section{Conclusions}

We found that UC was related to intestinal microbiota dysbiosis and we detected some specific core bacterial imbalances in UC. We also identified correlations between changes in certain metabolites and gut microbes. Sphingosine 1-phosphate was higher in UC patients and there were several microbiotas associated with it. Through pathway analysis, we found that sphingolipid metabolism was one of the most significantly increased pathways, so we predict that S1P will become a new target and provide new direction in the treatment of UC. Further research is needed to verify the effect of S1P in the ulcerative colitis in a mouse model.

\section{Conflict of interest}

None. 


\section{References:}

1. Becker C, Neurath MF, Wirtz S: The intestinal microbiota in inflammatory bowel disease. ILAR J, 2015; 56: 192-204

2. Ananthakrishnan AN: Epidemiology and risk factors for IBD. Nat Rev Gastroenterol Hepatol, 2015; 12: 205

3. Nee J, Feuerstein JD: Optimizing the care and health of women with inflammatory bowel disease. Gastroenterol Res Practice, 2015; 2015: 435820

4. Molodecky NA, Soon IS, Rabi DM et al: Increasing incidence and prevalence of the inflammatory bowel diseases with time, based on systematic review. Gastroenterology, 2015; 142: 46-54.e42

5. Wong SH, Ng SC: What can we learn from inflammatory bowel disease in developing countries? Curr Gastroenterol Rep, 2013; 15: 313

6. Hou JK, Abraham B, Elserag H: Dietary intake and risk of developing inflammatory bowel disease: A systematic review of the literature. Am J Gastroenterol, 2011; 106: 563

7. Danese S, Fiocchi C: Ulcerative colitis. N Engl J Med, 2011; 365: 1713-25

8. Hanauer SB: Inflammatory bowel disease: Epidemiology, pathogenesis, and therapeutic opportunities. Inflamm Bowel Dis, 2006; 12: S3-S9

9. Strober W, Fuss I, Mannon P: The fundamental basis of inflammatory bowel disease. J Clin Invest, 2007; 117: 514-21

10. Colombel JF, Watson AJM, Neurath MF: The 10 remaining mysteries of inflammatory bowel disease. Gut, 2008; 57: 429-33

11. Zeneng W, Elizabeth K, Bennett BJ et al: Gut flora metabolism of phosphatidylcholine promotes cardiovascular disease. Nature, 2011; 472: 57-63

12. Yue $\mathrm{C}$, Yang $\mathrm{X}$, Li J et al: Trimethylamine N-oxide prime NLRP3 inflammasome via inhibiting ATG16L1-induced autophagy in colonic epithelial cells. Biochem Biophys Res Commun, 2017; 490: 541-51

13. Seldin MM, Meng Y, Qi $\mathrm{H}$ et al: Trimethylamine $\mathrm{N}$-oxide promotes vascular inflammation through signaling of mitogen-activated protein kinase and nuclear factor-кB. J Am Heart Assoc, 2016; 5: e002767

14. Aze W, Teft WA, Morse BL et al: Trimethylamine-N-oxide: A novel biomarker for the identification of inflammatory bowel disease. Dig Dis Sci, 2015; 60: $3620-30$

15. Manichanh C, Rigottier-Gois L, Bonnaud E et al: Reduced diversity of faecal microbiota in Crohn's disease revealed by a metagenomic approach. Gut, 2006; 55: 205-11

16. Ott SJ, Musfeldt M, Wenderoth DF et al: Reduction in diversity of the colonic mucosa associated bacterial microflora in patients with active inflammatory bowel disease. Gut, 2004; 53: 685-93

17. Packey CD, Sartor RB: Commensal bacteria, traditional and opportunistic pathogens, dysbiosis and bacterial killing in inflammatory bowel diseases. Curr Opin Infect Dis, 2009; 22: 292-301

18. Li E, Hamm CM, Gulati AS et al: Inflammatory bowel diseases phenotype, C. difficile and NOD2 genotype are associated with shifts in human ileum associated microbial composition. PLoS One, 2012; 7: e26284

19. Pitcher MCL, Beatty ER, Cummings JH: The contribution of sulphate reducing bacteria and 5-aminosalicylic acid to faecal sulphide in patients with ulcerative colitis. Gut, 2000; 46: 64-72

20. Sutherland LR, Martin F, Greer S et al: 5-Aminosalicylic acid enema in the treatment of distal ulcerative colitis, proctosigmoiditis, and proctitis. Gastroenterology, 1987; 92: 1894-98

21. Org E, Blum Y, Kasela $\mathrm{S}$ et al: Relationships between gut microbiota, plasma metabolites, and metabolic syndrome traits in the METSIM cohort. Genome Biol, 2017; 18: 70

22. Kostic AD, Xavier RJ, Gevers D: The microbiome in inflammatory bowel disease: Current status and the future ahead. Gastroenterology, 2014; 146 1489-99
23. Machiels K, Joossens M, Sabino J et al: A decrease of the butyrate-producing species Roseburia hominis and Faecalibacterium prausnitzii defines dysbiosis in patients with ulcerative colitis. Gut, 2014; 63: 1275-83

24. Sartor RB: Microbial influences in inflammatory bowel diseases. Gastroenterology, 2008; 134: 577-94

25. Beaud D, Tailliez P, Anbamondoloni J: Genetic characterization of the betaglucuronidase enzyme from a human intestinal bacterium, Ruminococcus gnavus. Microbiology, 2005; 151: 2323-30

26. Shao T, Shao L, Li H et al: Combined signature of the fecal microbiome and metabolome in patients with gout. Front Microbiol, 2017; 8: 268

27. Segain JP, Raingeard de la Blétière $D$, Bourreille $A$ et al: Butyrate inhibits inflammatory responses through NFkappaB inhibition: Implications for Crohn's disease. Gut, 2000; 47: 397-403

28. Keller R, Ariöz C, Hansmeier N et al: The Escherichia coli envelope stress sensor CpxA responds to changes in lipid bilayer properties. Biochemistry, 2015; 54: 3670-76

29. Fontenelle LC, Feitosa MM, Severo JS et al: Thyroid function in human obesity: Underlying mechanisms. Horm Metab Res, 2016; 48: 787-94

30. Flamant F, Gauthier K, Richard S: Genetic investigation of thyroid hormone receptor function in the developing and adult brain. Curr Top Dev Biol, 2017; 125: 303-35

31. Apc T, Munhoz CD, Moriscot AS et al: S100A8/MYD88/NF-KB: A novel pathway involved in cardiomyocyte hypertrophy driven by thyroid hormone. J Mol Med, 2017; 95: 671-82

32. Proia RL, Hla T: Emerging biology of sphingosine-1-phosphate: its role in pathogenesis and therapy. J Clin Invest, 2015; 125: 1379-87

33. Roviezzo F, Di Lorenzo A, Bucci M et al: Sphingosine-1-phosphate/sphin gosine kinase pathway is involved in mouse airway hyperresponsiveness. Am J Respir Cell Mol Biol, 2007; 36: 757-62

34. Takeshita H, Kitano M, Iwasaki T et al: Sphingosine 1-phosphate (S1P)/S1P receptor 1 signaling regulates receptor activator of NF-KB ligand (RANKL) expression in rheumatoid arthritis. Biochem Biophys Res Commun, 2012; 419: 154-59

35. Van DR, Van HJ, Verzijl D et al: Sphingosine 1-phosphate receptor 1 and 3 are upregulated in multiple sclerosis lesions. Glia, 2010; 58: 1465-76

36. Roviezzo F, Brancaleone V, De Grutolla L et al: Sphingosine-1-phosphate modulates vascular permeability and cell recruitment in acute inflammation in vivo. J Pharmacol Exp Ther, 2011; 337: 830-37

37. Peyrinbiroulet L, Christopher R, Behan D, Lassen C: Modulation of sphingosine-1-phosphate in inflammatory bowel disease. Autoimmun Rev, 2017; 16: 495-503

38. Gevers D, Kugathasan S, Denson LA et al: The treatment-naive microbiome in new-onset Crohn's disease. Cell Host Microbe, 2014; 15: 382-92

39. Abbeele PVD, Belzer C, Goossens M et al: Butyrate-producing Clostridium cluster XIVa species specifically colonize mucins in an in vitro gut model. ISME J, 2013; 7: 949-61

40. Xiao M, Liu YG, Zou MC, Zou F: Sodium butyrate induces apoptosis of human colon cancer cells by modulating ERK and sphingosine kinase 2. Biomed Environ Sci, 2014; 27: 197-203

41. Thorkildsen LT, Nwosu FC, Avershina E et al: Dominant fecal microbiota in newly diagnosed untreated inflammatory bowel disease patients. Gastroenterol Res Prac, 2013; 2013: 636785

42. Chen L, Wang W, Zhou R et al: Characteristics of fecal and mucosa-associated microbiota in Chinese patients with inflammatory bowel disease. Medicine, 2014; 93: e51 